\title{
dspace.vutbr.cz
}

\section{Realization of first-order current-mode filters with low number of MOS transistors}

ERKAN YUCE, SHAHRAM MINAEI, NORBERT HERENCSAR, JAROSLAV KOTON

Citation: Journal of Circuits, Systems and Computers, Vol. 22, Iss. 1

ISSN: 0218-1266 (Print)

ISSN: 1793-6454 (Online)

DOI: http://dx.doi.org/10.1142/S0218126612500715

Accepted manuscript

Electronic version of an article published as Journal of Circuits, Systems and Computers, Volume 22, Issue 1, 2013, 1-14 pp. DOI 10.1142/S0218126612500715 (c) World Scientific Publishing Company, http://www.worldscientific.com/worldscinet/jcsc 


\title{
REALIZATION OF FIRST-ORDER CURRENT-MODE FILTERS WITH LOW NUMBER OF MOS TRANSISTORS
}

\author{
ERKAN YUCE \\ Pamukkale University, Department of Electrical and Electronics Engineering, \\ Denizli, Kinikli 20070, Turkey \\ erkanyuce@yahoo.com \\ SHAHRAM MINAEI ${ }^{*}$ \\ Dogus University, Department of Electronics and Communications Engineering, \\ Acibadem, Kadikoy 34722, Istanbul, Turkey \\ sminaei@dogus.edu.tr \\ NORBERT HERENCSAR \\ Brno University of Technology, Department of Telecommunications, \\ Purkynova 118, 61200 Brno, Czech Republic \\ herencsn@feec.vutbr.cz \\ JAROSLAV KOTON \\ Brno University of Technology, Department of Telecommunications, \\ Purkynova 118, 61200 Brno, Czech Republic \\ koton@feec.vutbr.cz \\ Received (Day Month Year) \\ Revised (Day Month Year) \\ Accepted (Day Month Year)
}

\begin{abstract}
In this paper, a new current-mode (CM) circuit for realizing all of the first-order filter responses is suggested. The proposed configuration contains low number of components, only two NMOS transistors both operating in saturation region, two capacitors and two resistors. Major advantages of the presented circuit are low voltage, low noise and high linearity. The proposed filter circuit can simultaneously provide both inverting and non-inverting first-order low-pass, high-pass and all-pass filter responses. Computer simulation results achieved through SPICE tool and experimental results are given as examples to demonstrate performance and effectiveness of the proposed topology.
\end{abstract}

Keywords: NMOS, first-order filter, current-mode.

\section{Introduction}

The use of current-mode (CM) active devices offers some important potential advantages called as higher usable gain, more reduced voltage excursion at sensitive nodes, greater linearity, less power consumption, wider bandwidth, better accuracy and larger dynamic range compared to that of voltage-mode counterparts for example operational amplifiers ${ }^{1-3}$. It is a well known fact that an all-pass filter (phase shifter) topology like previously reported first-order signal processing units ${ }^{4-18}$ finds wide 
Table 1. Comparison with previously published CM all-pass filters

\begin{tabular}{|l|l|l|l|l|l|l|}
\hline Properties & $\begin{array}{l}\text { Number of } \\
\text { transistors }\end{array}$ & $\begin{array}{l}\text { Number } \\
\text { of R/C }\end{array}$ & $\begin{array}{l}\text { Passive } \\
\text { component } \\
\text { matching } \\
\text { constraint }\end{array}$ & $\begin{array}{l}\text { Type of } \\
\text { response }\end{array}$ & Technology & $\begin{array}{l}\text { Power } \\
\text { supplies }\end{array}$ \\
\hline$[10]$ & 6 & $0 / 1$ & no & inverting & BJT & $\pm 1.5 \mathrm{~V}$ \\
\hline$[11]$ & 3 & $2 / 2$ & no & Non-inverting & $0.18 \mu \mathrm{m}$ & $+1.8 \mathrm{~V}$ \\
\hline$[12]$ & 7 & $0 / 1$ & no & Non-inverting & $0.35 \mu \mathrm{m}$ & $+3.3 \mathrm{~V}$ \\
\hline$[13]$ & 12 & $0 / 1$ & no & Non-inverting & BJT & $+3.0 \mathrm{~V}$ \\
\hline$[14]$ & 10 & $0 / 1$ & no & inverting & BJT & $\pm 5.0 \mathrm{~V}$ \\
\hline$[15]$ & $38^{*}$ & $2 / 1$ & yes & Both ** & $0.35 \mu \mathrm{m}$ & $\pm 2.5 \mathrm{~V}$ \\
\hline$[16]$ & 38 & $1 / 1$ & no & $\begin{array}{l}\text { Both } \\
\text { simultaneously }\end{array}$ & $0.5 \mu \mathrm{m}$ & $\pm 2.0 \mathrm{~V}$ \\
\hline$[17]$ & 32 & $1 / 1$ & no & Both *** & $0.25 \mu \mathrm{m}$ & $\pm 1.25 \mathrm{~V}$ \\
\hline This work & 2 & $2 / 2$ & yes & $\begin{array}{l}\text { Both } \\
\text { simultaneously }\end{array}$ & $0.13 \mu \mathrm{m}$ & $\pm 0.75 \mathrm{~V}$ \\
\hline
\end{tabular}

* Except four NMOS transistors used to obtain two tunable resistors

** There are two different circuits, one of which is inverting while other one is non-inverting.

*** Inverting response is obtained by RC-CR transformation.

application areas in analog signal processing systems, control engineering, etc. in order to shift phases of the signals while keeping their amplitudes unchanged. A first-order allpass filter requiring at least one capacitive component and one resistive component has a pole on the left half $s$ plane and a zero on the right half $s$ plane. The pole and zero has the same value in magnitude. The filters of ${ }^{4-9}$ use CMOS technology but operate in voltagemode (VM). On the other hand, the circuits in ${ }^{10,13,14}$ operate in CM but employ BJT technology. The CM filter of ${ }^{11}$, which is suitable for high frequency applications, contains three CMOS transistors, a floating resistor for biasing purpose and a large coupling capacitor for dc isolation of the load. The circuit reported in ${ }^{12}$ is a square-rootdomain CM filter which needs two identical bias currents. It is worth to note that a simple square-root-domain integrator needs more than 30 transistors ${ }^{12}$. Further, many phase shifters like circuits in ${ }^{15-18}$ using active building blocks such as second-generation current conveyors (CCIIs) have been reported in open literature. It should be noted that a CCII typically involves more than ten transistors.

In this paper, a novel $\mathrm{CM}$ circuit for simultaneously providing both inverting and non-inverting first-order low-pass, high-pass and all-pass filter responses is suggested. The developed filter structure includes a lower number of elements such as only two NMOS transistors, two capacitors and two resistors. The presented circuit enjoys low voltage, low noise and high linearity. However, it requires matching conditions for allpass responses. Simulation results and experimental measurements are included to verify the theory. Table 1 summarizes the advantages and disadvantages of the previously published CM all-pass phase filters as well as the proposed one.

\section{Proposed CM All-pass Filter}

The proposed CM topology for realizing various first-order filters is shown in Fig. 1. It can be seen that it employs an inverting amplifier with the voltage gain of $-\beta$. The employed inverting amplifier should possess high input and low output impedances ideally. Such an amplifier with unity gain in magnitude can be found in ${ }^{5}$ using two NMOS and three PMOS transistors or in ${ }^{6,8}$ using only two NMOS transistors with large 


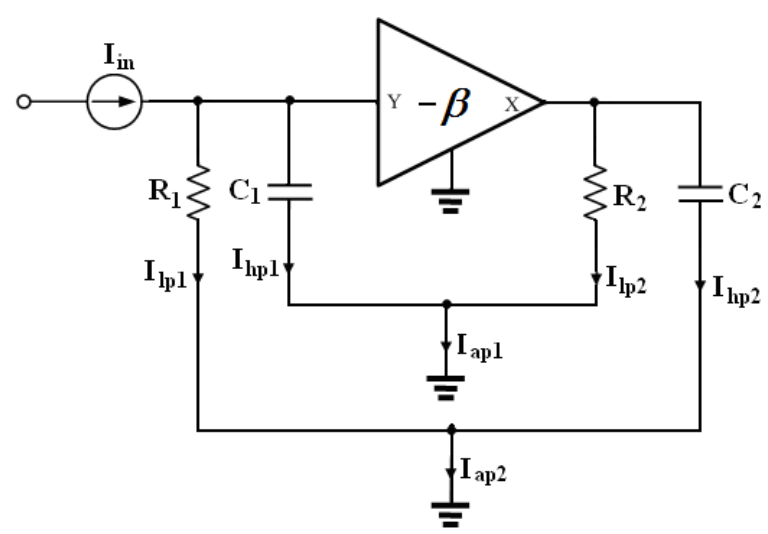

Fig. 1. Developed CM configuration for realizing first-order filters.

dimensions. Analysis of the proposed CM configuration of Fig. 1 gives the following responses:

i. Non-inverting low-pass transfer function (TF) and phase response

$$
\begin{aligned}
& \frac{I_{l p 1}}{I_{i n}}=\frac{1}{1+s C_{1} R_{1}} \\
& \varphi_{l p 1}(\omega)=-\tan ^{-1}\left(\omega C_{1} R_{1}\right)
\end{aligned}
$$

ii. Non-inverting high-pass TF and phase response

$$
\begin{aligned}
& \frac{I_{h p 1}}{I_{\text {in }}}=\frac{s C_{1} R_{1}}{1+s C_{1} R_{1}} \\
& \varphi_{h p 1}(\omega)=\frac{\pi}{2}-\tan ^{-1}\left(\omega C_{1} R_{1}\right)
\end{aligned}
$$

iii. Inverting low-pass $\mathrm{TF}$ and phase response

$$
\begin{gathered}
\frac{I_{l p 2}}{I_{\text {in }}}=-\frac{\beta \frac{R_{1}}{R_{2}}}{1+s C_{1} R_{1}} \\
\varphi_{l p 2}(\omega)=\pi-\tan ^{-1}\left(\omega C_{1} R_{1}\right)
\end{gathered}
$$

iv. Inverting high-pass $\mathrm{TF}$ and phase response

$$
\begin{gathered}
\frac{I_{h p 2}}{I_{i n}}=-\frac{s \beta C_{2} R_{1}}{1+s C_{1} R_{1}} \\
\varphi_{h p 2}(\omega)=-\frac{\pi}{2}-\tan ^{-1}\left(\omega C_{1} R_{1}\right)
\end{gathered}
$$


Here, the pole frequency of the filter from Fig. 1 is found as $\omega_{0}=1 /\left(C_{1} R_{1}\right)$. Further, if $\beta=1$ as for the inverting amplifiers in ${ }^{5,6}$, the following responses can be obtained:

v. Inverting all-pass TF and phase response if $R_{2}=R_{1}$ as

$$
\begin{aligned}
& \frac{I_{a p 1}}{I_{\text {in }}}=\frac{I_{h p 1}}{I_{\text {in }}}+\frac{I_{l p 2}}{I_{\text {in }}}=-\frac{1-s C_{1} R_{1}}{1+s C_{1} R_{1}} \\
& \varphi_{\text {ap } 1}(\omega)=\pi-2 \tan ^{-1}\left(\omega C_{1} R_{1}\right)
\end{aligned}
$$

vi. Non-inverting all-pass TF and phase response if $C_{2}=C_{1}$ as

$$
\begin{aligned}
& \frac{I_{a p 2}}{I_{i n}}=\frac{I_{l p 1}}{I_{i n}}+\frac{I_{h p 2}}{I_{i n}}=\frac{1-s C_{1} R_{1}}{1+s C_{1} R_{1}} \\
& \varphi_{a p 2}(\omega)=-2 \tan ^{-1}\left(\omega C_{1} R_{1}\right)
\end{aligned}
$$

It should be noted that the all-pass filters of ${ }^{4-15,17,18}$ can realize only one all-pass response. In order to obtain output currents at high-output-impedance terminals from the structure in Fig. 1, a current buffer is required for each current. For this purpose a current follower (CF) ${ }^{19}$ can be a good choice. However, a CF has an input parasitic resistance denoted by $R_{p}$. The output of the developed inverting all-pass filter connected to the input of a CF is shown in Fig. 2. Considering the required conditions for the inverting all-pass response as $R_{1}=R_{2}$ and $\beta=1$, the corresponding gain and phase responses are computed as

$$
\frac{I_{a p 1}}{I_{i n}}=-\frac{1-s C_{1} R_{1}}{1+\frac{R_{p}}{R_{1}}+s C_{1}\left(R_{1}+3 R_{p}\right)}
$$

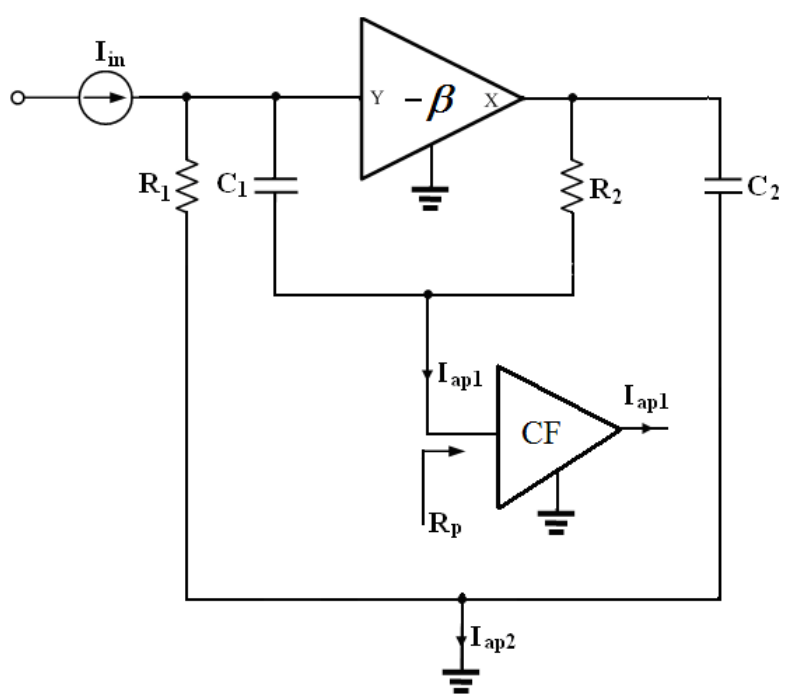

Fig. 2. The output of the suggested all-pass filter connected to the input of a CF. 


$$
\varphi_{a p 1}(\omega)=\pi-\tan ^{-1}\left(\omega C_{1} R_{1}\right)-\tan ^{-1}\left(\frac{\omega C_{1}\left(R_{1}+3 R_{p}\right)}{1+\frac{R_{p}}{R_{1}}}\right)
$$

From (7), one should select $R_{1} \gg 3 R_{p}$ to prevent the loading effect.

\section{Parasitic effects}

The parasitic of the employed amplifier can be considered as parallel capacitance and resistance at its input terminal $\left(R_{y}|| C_{y}\right)$ and output resistance $\left(R_{o}\right)$ in series at its output terminal as shown in Fig. 3. Since $R_{y}$ and $C_{y}$ appears in parallel with externally connected $R_{1}$ and $C_{1}$, their effects can be neglected selecting $R_{1}>>R y$ and $C_{y}<<C_{1}$. Considering the output resistance $R_{o}$ of the inverting amplifier, while the current responses $\mathrm{I}_{l p 1}$ and $\mathrm{I}_{h p 1}$ remain undisturbed, $I_{l p 2}$ and $I_{h p 2}$ and their corresponding phase responses convert to

$$
\begin{aligned}
& \frac{I_{l p 2}}{I_{\text {in }}}=-\frac{\beta R_{1}}{\left(1+s C_{1} R_{1}\right)\left(R_{2}+R_{o}+s C_{2} R_{2} R_{o}\right)} \\
& \varphi_{l p 2}(\omega)=\pi-\tan ^{-1}\left(\omega C_{1} R_{1}\right)-\tan ^{-1}\left(\frac{\omega C_{2} R_{2} R_{o}}{R_{2}+R_{o}}\right) \\
& \frac{I_{h p 2}}{I_{\text {in }}}=-\frac{\beta s C_{2} R_{1} R_{2}}{\left(1+s C_{1} R_{1}\right)\left(R_{2}+R_{o}+s C_{2} R_{2} R_{o}\right)} \\
& \varphi_{l p 2}(\omega)=-\frac{\pi}{2}-\tan ^{-1}\left(\omega C_{1} R_{1}\right)-\tan ^{-1}\left(\frac{\omega C_{2} R_{2} R_{o}}{R_{2}+R_{o}}\right)
\end{aligned}
$$

It can be seen that a second pole appears in the TF of the filter due to the nonzero output resistance of the amplifier as

$$
\omega_{p 2}=\frac{R_{2}+R_{o}}{C_{2} R_{2} R_{o}}=\frac{1}{C_{2} R_{o}}+\frac{1}{C_{2} R_{2}}
$$

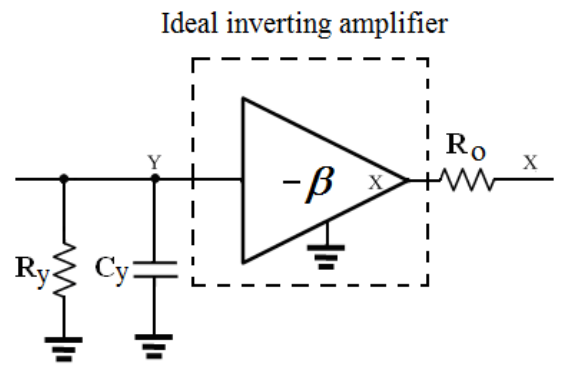

Fig. 3. Inverting amplifier with parasitic impedances 
Thus one should select $R_{o}<<R_{2}$ in order to neglect the effect of the second pole. Similarly, for the all-pass responses we can obtain

$$
\begin{gathered}
\frac{I_{\text {lap } 1}}{I_{\text {in }}}=-\frac{R_{1}\left(\beta-s C_{1}\left(R_{2}+R_{o}\right)-s^{2} C_{1} C_{2} R_{2} R_{o}\right)}{\left(1+s C_{1} R_{1}\right)\left(R_{2}+R_{o}+s C_{2} R_{2} R_{o}\right)} \\
\varphi_{a p 1}(\omega)=\pi-\tan ^{-1}\left(\frac{\omega C_{1}\left(R_{2}+R_{o}\right)}{\beta+\omega^{2} C_{1} C_{2} R_{2} R_{o}}\right)-\tan ^{-1}\left(\omega C_{1} R_{1}\right)-\tan ^{-1}\left(\frac{\omega C_{2} R_{2} R_{o}}{R_{2}+R_{o}}\right) \\
\frac{I_{\text {lap } 2}}{I_{\text {in }}}=\frac{R_{2}+R_{o}-s C_{2} R_{2}\left(\beta R_{1}-R_{o}\right)}{\left(1+s C_{1} R_{1}\right)\left(R_{2}+R_{o}+s C_{2} R_{2} R_{o}\right)} \\
\varphi_{a p 2}(\omega)=-\tan ^{-1}\left(\frac{\omega C_{2} R_{2}\left(\beta R_{1}-R_{o}\right)}{R_{2}+R_{o}}\right)-\tan ^{-1}\left(\omega \mathrm{C}_{1} \mathrm{R}_{1}\right)-\tan ^{-1}\left(\frac{\omega C_{2} R_{2} R_{o}}{R_{2}+R_{o}}\right)
\end{gathered}
$$

Selecting $R_{o}<<R_{2}$ with $R_{2}=\beta R_{1}$ and $C_{1}=\beta C_{2}$ in (11a) and (12a) one can obtain the ideal all-pass responses (5a) and (6a), respectively. Note that the proposed all-pass filter having no capacitor connected in series to the $\mathrm{X}$ terminal of the inverting amplifier; accordingly, it can be worked at higher frequencies ${ }^{20}$.

\section{Noise analysis}

The inverting amplifier used in the structure of the proposed CM all-pass filter can be implemented with two NMOS transistors as shown in Fig. $4 \mathrm{a}^{8}$. The gain of the amplifier is found as

$$
\frac{V_{O}}{V_{\text {in }}}=-\beta=-\sqrt{\frac{(W / L)_{1}}{(W / L)_{2}}}
$$

where $(W / L)_{i}$ is the ratio of the channel width to channel length of the $i$-th $(i=1,2)$ transistor.

The inverting amplifier including noise sources of the transistors is shown in Fig. 4b. The noise density sources of the transistors can be represented as:

$$
\begin{aligned}
& \overline{d V_{1}^{2}}=4 k T R_{\text {eff } 1} d f \\
& \overline{d V_{2}^{2}}=4 k T R_{\text {eff } 2} d f
\end{aligned}
$$

Here, $R_{\text {effi }}$ is the effective thermal noise resistance of the $i$-th transistor given as

$$
R_{e f f i}=\frac{\frac{2}{3}}{g_{m i}}+R_{G i}+R_{B i}(n-1)^{2}
$$



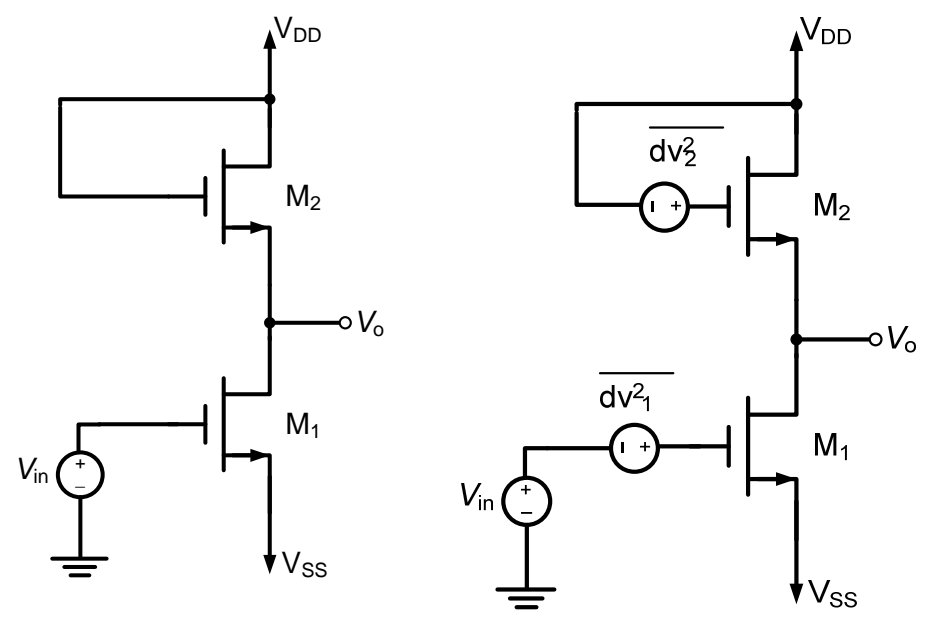

Fig. 4. (a) NMOS-based inverting amplifier (b) Noise model.

In (15), $\frac{2 / 3}{g_{m}}$ represents the channel noise effect, $R_{G}$ is the poly gate resistor and $R_{B}$ is the substrate resistance ${ }^{21}$. The substrate resistance is multiplied a factor of $(n-1)$ which is equal to the ratio of the bulk transconductance to the transconductance of the transistor, i.e. $\frac{g_{m b}}{g_{m}}$.

The total output current noise density of the inverting amplifier can be given as

$$
\overline{d i_{\text {out }}^{2}}=g_{m 1} 2 \overline{d V_{1}^{2}}+g_{m 2} 2 \overline{d V_{2}^{2}}
$$

Thus the equivalent input noise density of the amplifier is found as

$$
\overline{d V_{i e q}^{2}}=\overline{d V_{1}^{2}}+\frac{g_{m 2}^{2}}{g_{m 1}^{2}} \overline{d V_{2}^{2}}=\overline{d V_{1}^{2}}+\frac{1}{\beta^{2}} \overline{d V_{2}^{2}}
$$

Considering matched transistors i.e. $g_{m 1} \cong g_{m 2}$ and consequently $\beta=1$ the total equivalent input noise density is $\overline{d V_{i e q}^{2}} \cong 2 \overline{d V_{1}^{2}}$.

For the all-pass filter of Fig. 1, there are additional thermal noises due to the external resistors $R_{1}$ and $R_{2}$. Thus, the total equivalent input noise of the proposed CM all-pass filter can be given as:

$$
\overline{d V_{i e q-a l l p a s s}^{2}}=4 k T R_{1} d f+2 \overline{d V_{1}^{2}}+\frac{1}{\beta^{2}} 4 k T R_{2} d f
$$

From (18), an equivalent input resistance $\left(R_{\text {ieq }}\right)$ for noise calculation purpose can be defined as: 


$$
R_{\text {ieq-allpass }}=R_{1}+2 R_{\text {eff } 1}+\frac{1}{\beta^{2}} R_{2}
$$

The bandwidth (BW) of the equivalent input noise density is limited by the external capacitor $C_{1}$. Thus the integrated noise of this resistor-capacitor combination (or all-pass filter) is found by taking the integral over all frequencies as

$$
\overline{V_{i e q}^{2}}=\int_{0}^{\infty} \frac{\overline{d V_{i e q-a l l p a s s}^{2}}}{1+\left(\frac{f}{B W}\right)^{2}}=4 k T R_{i e q} B W \frac{\pi}{2}
$$

where

$$
B W=\frac{1}{2 \pi R_{i e q} C_{1}}
$$

Substituting (21) into (20), the integrated noise is simply found as $k T / C_{1}$. For wideband systems the integrated noise is important, thus to reduce the noise we have to select larger capacitances which obviously will increase the power consumption ${ }^{21}$.

\section{Simulation and experimental results}

In this section, simulation results of the proposed first-order all-pass filter in Fig. 1 based on $0.13 \mu \mathrm{m}$ IBM MOS technology parameters ${ }^{22}$ with $\pm 0.75 \mathrm{~V}$ dc power supply voltages are demonstrated. The aspect ratios of both NMOS transistors of inverting amplifier in Fig. $4{ }^{6}$ are chosen as $104 \mu \mathrm{m} / 0.13 \mu \mathrm{m}$. The aspect ratio of the transistors are selected large to prevent loading effect. The non-ideality parameters of the structure given in Fig. 4 are found as $\beta=0.852, R_{o}=13.66 \Omega, C_{y}=221 \mathrm{fF}, R_{y} \approx \infty$. Passive components of the all-pass filter depicted in Fig. 1 are taken as $R_{1}=R_{2}=12 \mathrm{k} \Omega$ and $C_{1}=C_{2}=10 \mathrm{pF}$ to obtain the resonance frequency of $f_{\mathrm{o}} \cong 1.32 \mathrm{MHz}$. For this purpose, ideal and simulated magnitude and phase responses of the all-pass filter, $I_{a p 1}$ and $I_{a p 2}$, are shown in Figs. 5 and 6, respectively. Time domain responses of $I_{a p 1}$ and $I_{a p 2}$ are demonstrated in Fig. 7. Total Harmonic Distortion (THD) variations versus amplitudes of peak values of input current are given in Table 2, where at the outputs of $I_{a p 1}$ and $I_{a p 2}$. $20 \Omega$ resistors are connected to simulate the effect of the current. Similarly, input and output noise variations against frequency for $I_{a p 1}$ and $I_{a p 2}$ are tabulated in Tables 3 and 4, respectively.

Monte Carlo analysis of the introduced all-pass filter with $20 \%$ variations of $C=10 \mathrm{pF}$ is achieved after a hundred runs. In addition, the variations of the magnitude of all-pass filter at $1.32 \mathrm{MHz}$ are demonstrated in Fig. 8. The magnitudes of $I_{a p 1}$ and $I_{a p 2}$ with respect to frequency are drawn in Fig. 9.

In order to confirm the simulation results, the behavior of the $I_{a p 1}$ response of the proposed all-pass filter has also been verified by experimental measurements using network-spectrum analyzer Agilent 4395A, function generator Agilent 33521A, and oscilloscope Agilent DSOX2014A. In measurements the readily available array transistors CD4007UB ${ }^{23}$ by Texas Instruments with $\pm 15 \mathrm{~V}$ dc supply voltages have been used. To perform the measurements of the proposed current-mode filter, the circuit was extended by voltage-to-current and current-to-voltage converters realized by OPA860 ICs ${ }^{24}$ by Texas Instruments with dc power supply voltages equal to $\pm 5 \mathrm{~V}$ as it is shown in ${ }^{25}$. 
Since the OPA 860 generally behaves as CCII+, the measured voltage compared to the $I_{a p 1}$ is shifted in the phase by $180^{\circ}$. The experiments have been performed with $R_{1}=R_{2}=1.2 \mathrm{k} \Omega$ and $C_{1}=C_{2}=100 \mathrm{pF}$ and the results are shown in Fig. 10. In this case the $90^{\circ}$ phase shift is at $f_{\mathrm{o}} \cong 1.26 \mathrm{MHz}$, which is very close to the theoretical value of $f_{\mathrm{o}} \cong 1.3263 \mathrm{MHz}$. The time-domain response of the measured $I_{a p 1}$ response of the all-pass filter is shown in Fig. 11 in which a sine-wave input of $1 \mathrm{~V}$ peak-to-peak and frequency of $1.26 \mathrm{MHz}$ was applied to the filter. From Fig. 11 it can be seen that the phase shift in the $I_{a p 1}$ output against the input is $91^{\circ}$.

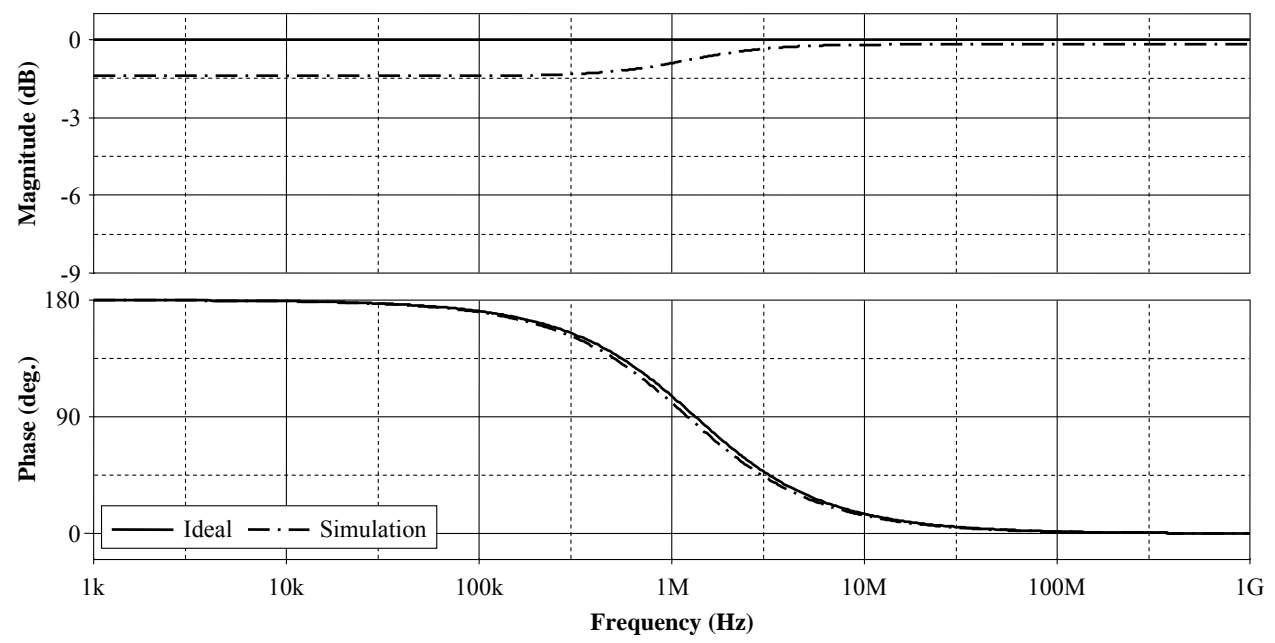

Fig. 5. Simulated magnitude and phase responses of the $I_{a p 1}$.
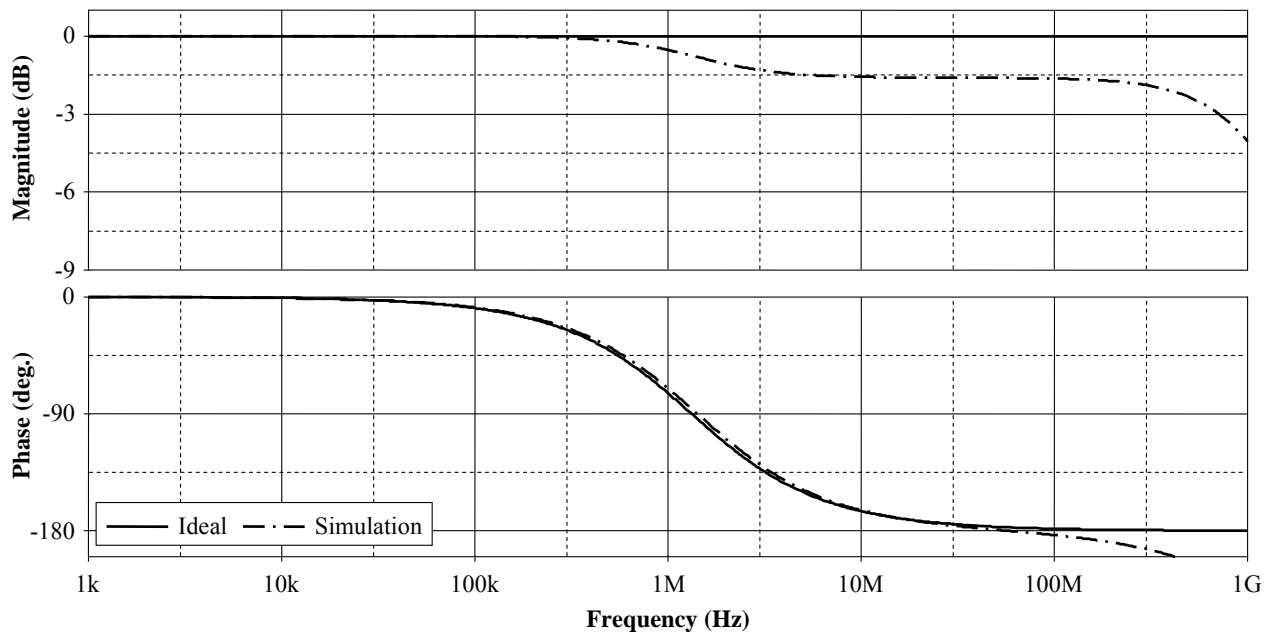

Fig. 6. Simulated magnitude and phase responses of the $I_{a p 2}$. 

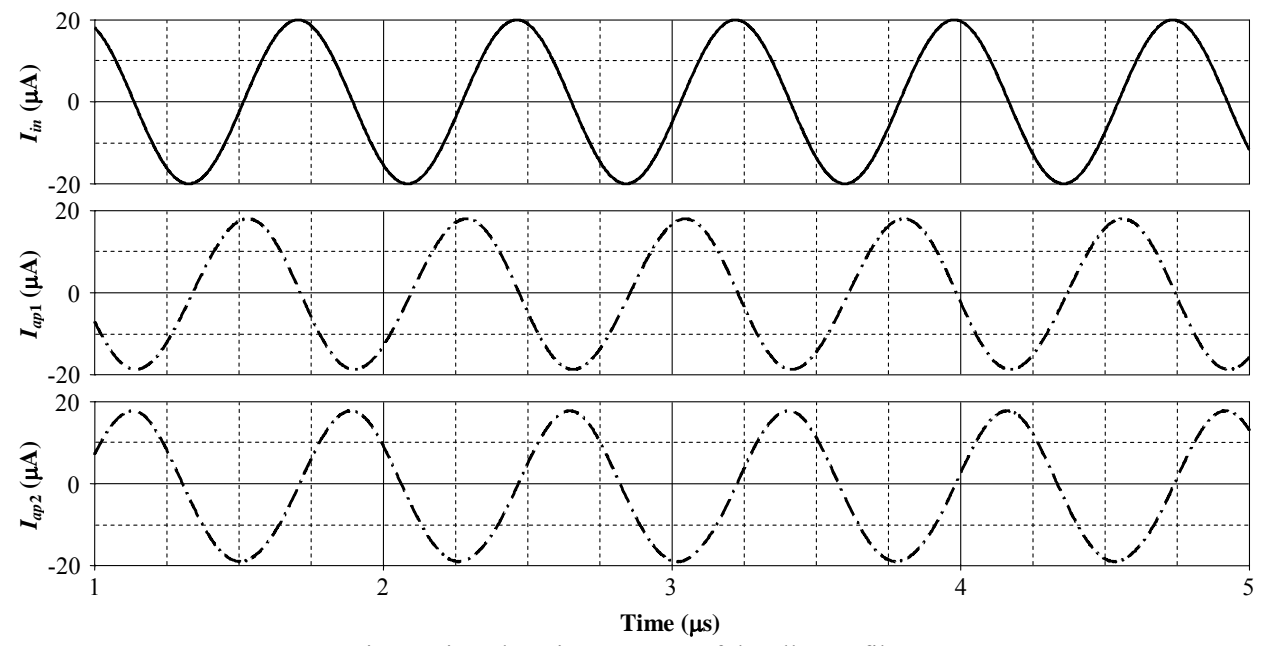

Fig. 7. Time domain responses of the all-pass filter.

Table 2. THD variations of all-pass responses of the filter in Fig. 1 at $1.32 \mathrm{MHz}$.

\begin{tabular}{||l||l||l||}
\hline \hline Magnitude of input current $(\mu \mathrm{A})$ & $I_{a p 2, \text { THD }(\%)}$ \\
\multicolumn{1}{|l||}{} & \multicolumn{1}{|||}{} \\
\hline \hline 1 & 0.379 & 0.628 \\
\hline 5 & 0.582 & 1.242 \\
\hline 10 & 1.119 & 2.306 \\
\hline 15 & 1.609 & 3.275 \\
\hline 20 & 2.046 & 4.072 \\
\hline 25 & 2.353 & 4.684 \\
\hline 30 & 2.581 & 5.192 \\
\hline 35 & 2.782 & 5.639 \\
\hline 40 & 2.955 & 6.050 \\
\hline 45 & 3.114 & 6.490 \\
\hline 50 & 3.232 & 7.103 \\
\hline
\end{tabular}

Table 3. Input and output noises of the all-pass filter in Fig. $1\left(I_{a p 1}\right)$ against frequency.

\begin{tabular}{||l||l||l||}
\hline Frequency $(\mathrm{Hz})$ & Output noise $(\mathrm{pV} / \sqrt{ } \mathrm{Hz})$ & Input noise $(\mathrm{pA} / \sqrt{\mathrm{Hz}})$ \\
\hline \hline $1 \times 10^{3}$ & 575.64 & 33.81 \\
\hline \hline $1 \times 10^{4}$ & 575.64 & 33.81 \\
\hline \hline $1 \times 10^{5}$ & 575.63 & 33.78 \\
\hline \hline $1 \times 10^{6}$ & 575.04 & 31.98 \\
\hline \hline $1 \times 10^{7}$ & 574.04 & 29.54 \\
\hline \hline \hline $1 \times 10^{8}$ & 574.02 & 29.48 \\
\hline \hline
\end{tabular}


Table 4. Input and output noises of the all-pass filter in Fig. $1\left(I_{a p 2}\right)$ against frequency.

\begin{tabular}{||l||l||l||}
\hline \hline Frequency $(\mathrm{Hz})$ & Output noise $(\mathrm{pV} / \sqrt{\mathrm{Hz}})$ & Input noise $(\mathrm{pA} / \sqrt{\mathrm{Hz}})$ \\
\hline \hline \hline $1 \times 10^{3}$ & 575.77 & 28.79 \\
\hline \hline $1 \times 10^{4}$ & 575.77 & 28.79 \\
\hline \hline $1 \times 10^{5}$ & 575.77 & 28.82 \\
\hline \hline $1 \times 10^{6}$ & 575.72 & 30.67 \\
\hline \hline $1 \times 10^{7}$ & 575.59 & 34.69 \\
\hline \hline $1 \times 10^{8}$ & 572.45 & 35.11 \\
\hline \hline
\end{tabular}
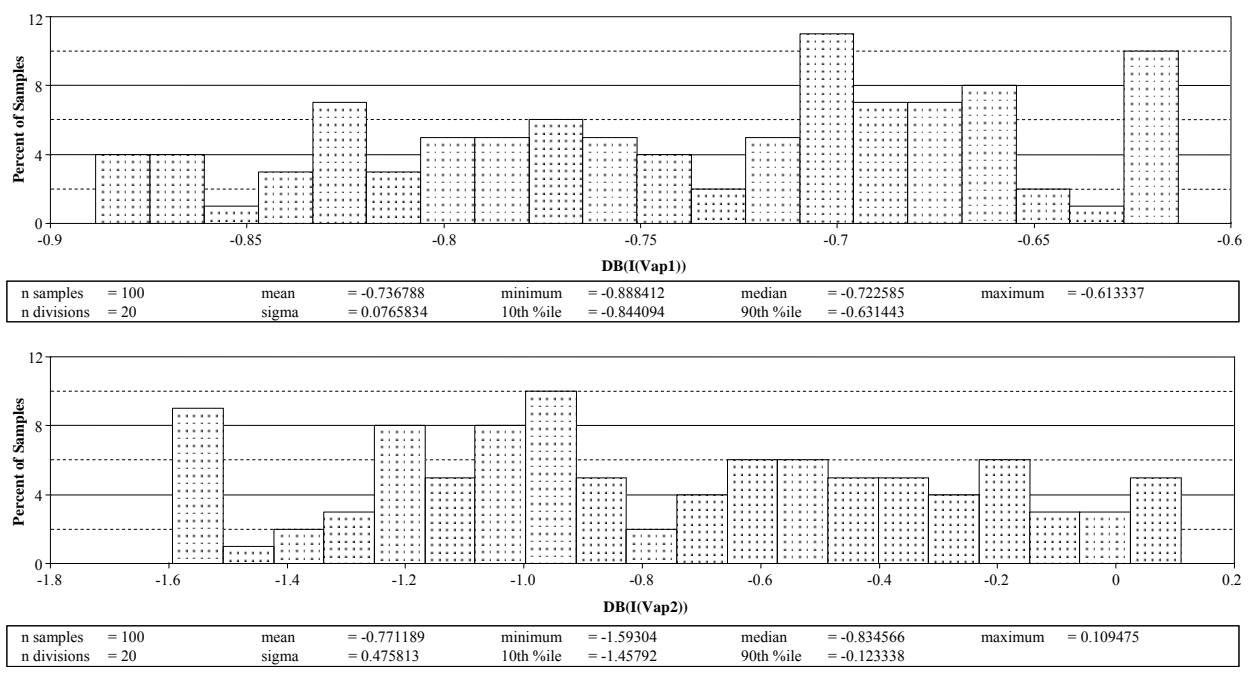

Fig. 8. Variations of the magnitude of all-pass filter at $1.32 \mathrm{MHz}$.
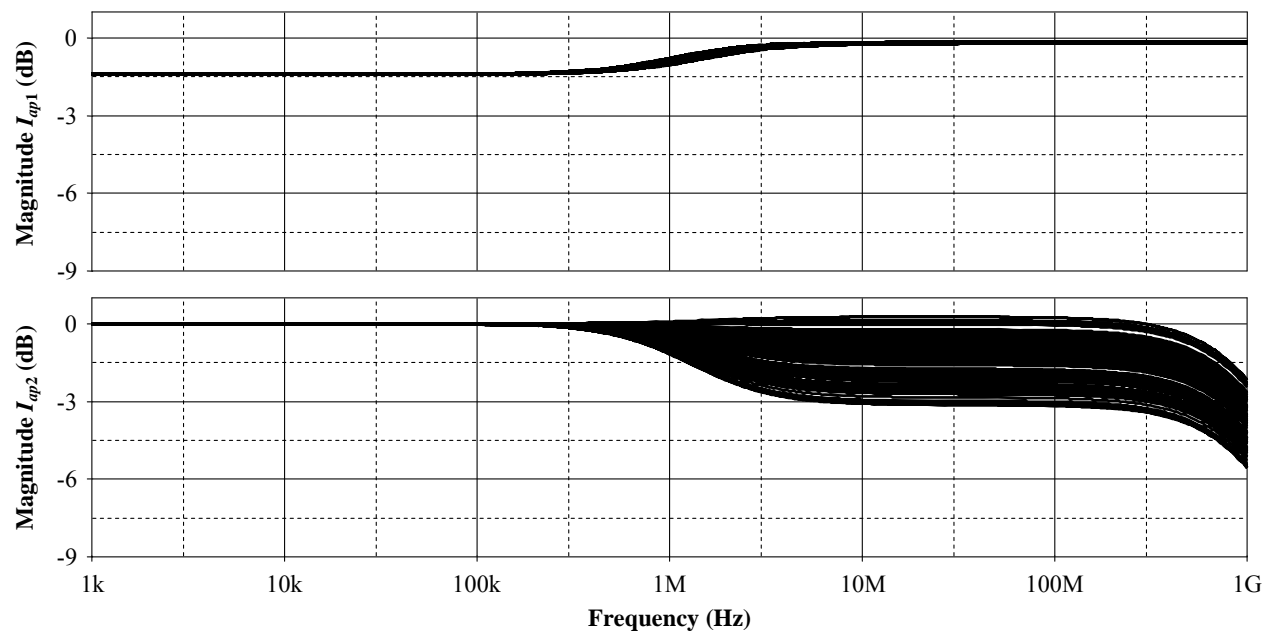

Figure 9. The magnitudes of $I_{a p 1}$ and $I_{a p 2}$ with respect to frequency. 


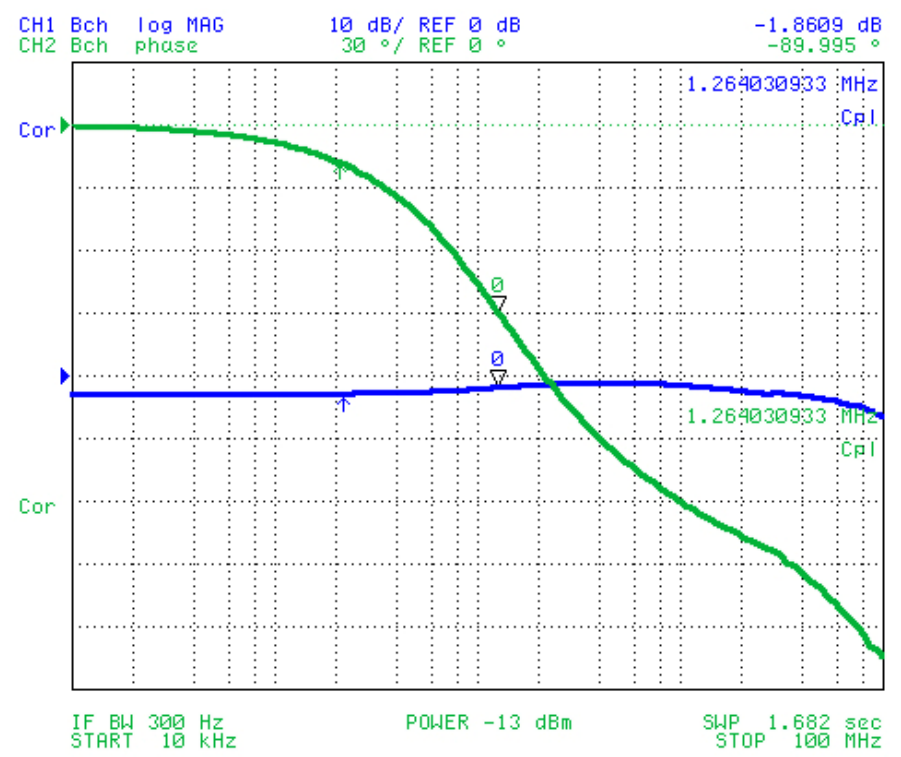

Fig. 10. Measured magnitude and phase responses of the $I_{a p 1}$.

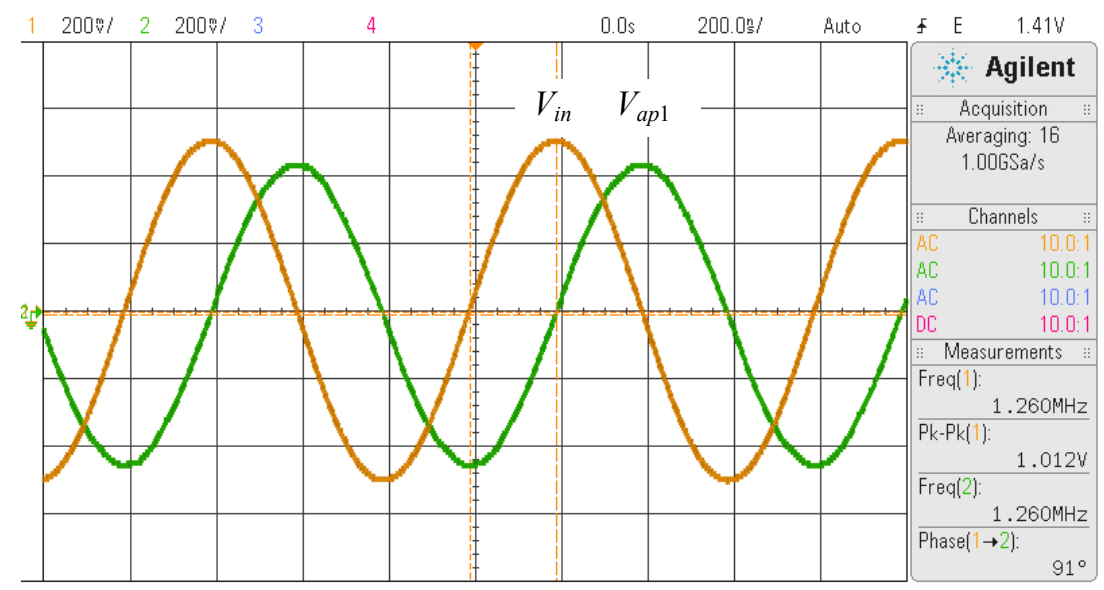

Fig. 11. Measured time domain response of the $I_{a p 1}$.

It is observed from Figs. 5-7, 10, and 11 that the simulation and experimental results are in close proximity with the ideal ones as expected. Nevertheless, the discrepancy among ideal, simulation and experimental results fundamentally arises from nonidealities of NMOS transistors.

\section{Conclusion}

A new CM all-pass filter structure for simultaneously realizing inverting/ noninverting first-order low-pass, high-pass and all-pass filter responses is developed in this paper. The suggested first-order filter consists of a lower number of components for example two NMOS transistors both in saturation region, two capacitors and two resistors. Major advantages of the proposed configuration are low voltage, low noise and 
high linearity. Nevertheless, it needs matching constraints for all-pass characteristics. Simulation results accomplished via SPICE and experimental tests confirm the theoretical ones well as expected.

\section{Acknowledgment}

The research described in the paper was supported by the following projects: P102/11/P489, P102/10/P561, P102/09/1681, FEKT-S-11-15, and project SIX CZ.1.05/2.1.00/03.0072 from the operational program Research and Development for Innovation.

Authors also wish to thank the anonymous reviewers for their useful and constructive comments that helped to improve the paper.

\section{References}

1. B. Wilson, Trends in current conveyor and current-mode amplifier design, International Journal of Electronics 73 (1992) 573-583.

2. C. Toumazou, F. J. Lidgey and D. G. Haigh, Analog IC design: the current-mode approach, London: Peter Peregrinus, 1990.

3. G. W. Roberts, and A. S. Sedra, "All current-mode frequency selective circuits," Electronics Letters 25 (1989) 759-761.

4. B. J. Maundy and P. Aronhime, A novel CMOS first order all pass filter, International Journal of Electronics 89 (2002) 739-743.

5. A. Toker, and S. Ozoguz, Tunable allpass filter for low voltage operation, Electronics Letters 39 (2003) 175-176.

6. E. Yuce, S. Minaei, A novel phase shifter using two NMOS transistors and passive elements, Analog Integrated Circuits and Signal Processing (ALOG) 62 (2010) 77-81.

7. E. Yuce, A novel CMOS-based voltage-mode first-order phase shifter employing a grounded capacitor, Circuits, Systems and Signal Processing 29 (2010) 235-245.

8. S. Minaei and E. Yuce, High input impedance NMOS-based phase shifter with minimum number of passive elements, Circuits, Systems and Signal Processing 31 (2012) 51-60.

9. E. Yuce and S. Minaei, Derivation of low-power first-order low-pass, high-pass and allpass filters, Analog Integrated Circuits and Signal Processing (ALOG) 70 (2012) 151156.

10. R. Arslanalp and E. Yuce, A BJT technology-based current-mode tunable all-pass filter, Microelectronics Journal 40 (2009) 921-927.

11. A. Mohammadi, A. Ayatollahi and A. Abrishamifar, A new CMOS all-pass phase shifter for phased array systems, IEICE Electronics Express 6 (2009) 504-510.

12. S. Ozoguz, T. M. Abdelrahman, A. S. Elwakil, Novel approximate square-root domain all-pass filter with application to multiphase oscillators, Analog Integrated Circuits and Signal Processing (ALOG) 46 (2006) 297-301.

13. R. Arslanalp, A. T. Tola and E. Yuce, Novel resistorless first-order current-mode universal filter employing a grounded capacitor, Radioengineering 20 (2011) 656-665.

14. R. Arslanalp, A. T. Tola and E. Yuce, Fully controllable first order current mode universal filter composed of BJTs and a grounded capacitor, Elektronika Ir Elektrotechnika 6 (2011) 69-72. 
15. B. Metin, K. Pal and O. Cicekoglu, All-pass filter for rich cascadability options easy IC implementation and tunability, International Journal of Electronics 94 (2007) 10371045.

16. S. M. Al-Shahrani, CMOS wideband auto-tuning phase shifter circuit, Electronics Letters 43 (2007) 804-806.

17. S. Minaei and E. Yuce, All grounded passive elements current-mode all-pass filter, Journal of Circuits, Systems and Computers 18 (2009) 31-43.

18. S. Minaei and E. Yuce, Novel voltage-mode all-pass filter based on using DVCCs, Circuits, Systems and Signal Processing 29 (2010) 391-402.

19. W. Tangsrirat and T. Pukkalanun, Digitally programmable current follower and its applications, International Journal of Electronics and Communications (AEU) 63 (2009) 416-422.

20. E. Yuce and S. Minaei, Universal current-mode filters and parasitic impedance effects on the filter performances, International Journal of Circuit Theory and Applications 36 (2008) 161-171.

21. W. M. C. Sansen, Analog Design Essentials, Springer, pp. 117-131, 2006.

22. http://www.mosis.com/cgi-bin/cgiwrap/umosis/swp/params/ibm-013/t97f_8hp_5lmparams.txt

23. Datasheet CD4007UB - CMOS Dual Complementary Pair Plus Inverter. Texas Instruments, SCHS018C-Rev. September 2003.

24. Datasheet OPA860 - Wide Bandwidth Operational Transconductance Amplifier (OTA) and Buffer. Texas Instruments, SBOS331C-June 2005-Rev. August 2008.

25. J. Koton, N. Herencsar and K. Vrba, Electronically tunable pure current-mode low-pass filter, In Proceedings of the 6th International Conference on Electrical and Electronics Engineering (ELECO 2009), Bursa, Turkey, pp. 260-262, 2009. 